Materials and Methods 141 surgical procedures were performed on children 3 - 16 years old in 2006-2016. The purpose of surgery was eliminating the instability of hip (GMFCS 1-3) and relieving pain (GMFCS 4-5).

Triple pelvic osteotomy (TPO) was performed in 5 cases on 5 patients $6-15$ years old; TPO combined with varus-detorsion femoral osteotomy (VDFO) - in 8 cases on 6 patients 711 years old. TPO, shortening VDFO and open head reduction were performed in 6 cases on 5 patients 7-13 years old who had hip dislocations.

VDFO combined with Salter's pelvic osteotomy were performed in 4 cases (4-6 years old).

Adductor myotomy was performed in 99 cases (43 patients bilaterally), it was combined with other soft tissue releases in the lower limbs in 32 cases.

Results Hip stabilization was achieved in all cases using TPO and TPO combined with

VDFO Reoperation was necessary in 3 cases after VDFO. The best results after the soft tissue releases were obtained when the Reimer's migration index was $<30 \%$, although in some cases joint stabilization was achieved after the migration index was $>80 \%$. For this group of patients femoral osteotomy was required in 12 cases, femoral and pelvic osteotomies - in 3 cases.

Conclusions Individual approach with a clear understanding goals of hip surgery and adherence to the principle 'one-stage and multilevel correction' are necessary in the treatment of children with CP.

When the Reimer's migration index is $<30 \%$ the soft tissues release can provide hip stability, in the most severe cases combination of pelvic and femur osteotomies leads to a better result.

\section{P39 PHOSPHATE: THE FORGOTTEN BROTHER - A CASE OF HYPOPHOSPHATAEMIC RICKETS}

${ }^{1}$ Eleanor Burke* ${ }^{1,2}$ Ciara McDonnell. 'Department of Paediatric Endocrinology, Temple Street Children's University Hospital, Dublin, Ireland; ${ }^{2}$ Discipline of Paediatrics, University of Dublin, Trinity College, Dublin, Ireland

10.1136/archdischild-2019-epa.394

Background Hypophosphataemic rickets is a rare metabolic bone disease. The X-linked [PHEX mutation] is the commonest variant [incidence of 1:20,000 newborns] and results in persistent elevation in Fibroblast Growth Factor (FGF)-23 levels which cause increased urinary phosphate excretion and corresponding hypophosphataemia. The phenotype is of bowed legs, short stature, reduced mobility and dental abscesses. Until recently, the standard of care was replacement of phosphate and activated vitamin $\mathrm{D}$ but novel anti-FGF-23 Ab [Burosu$\mathrm{mab}$ ] has improved quality of life and long-term outcomes for patients.

Case Description An 18 month-old boy in good general health, presented with pronounced bowing of his legs since weightbearing had commenced, associated with reduced growth velocity, flat feet and unsteady gait. Biochemisty was normal apart from low phosphate and raised alkaline phosphatase. Plain radiograph of the lower limbs demonstrated fraying of the distal medial $1 / 3^{\text {rd }}$ of the metaphyses of the femora and tibiae bilaterally. This characteristic appearance led to suspicion of hypophosphataemic rickets confirmed by elevated FGF-23 levels and genetic confirmation of a de novo mutation in the PHEX gene.
This patient was initiated on oral phosphate and one-alpha vitamin $\mathrm{D}$ replacement resulting in healing of rickets and improvement of growth. However, his fatigue remains evident and is associated with poor exercise tolerance and persistence dental issues. Intervention of physiotherapy [posture and strengthening] and orthopaedics [epiphysiodesis] have improved gait, straightening of legs and growth. However, patients in the UK have demonstrated normalisation of biochemistry, improvement of muscle strength and improved long-term mobility with weekly subcutaneous Burosumab injections which is awaiting approval in Ireland.

Discussion/Learning Points Rickets in Caucasian children in Ireland is usually genetic in origin.

Prompt identification of cause allows for early intervention and reduced skeletal deformity. Burosumab [anti-FGF23], the first approved targeted medication for X-linked hypo-phosphataemic rickets was FDA approved in 2018 and is awaiting approval in Ireland. Patients in clinical trials have demonstrated normalisation of biochemistry, improved muscle strength and long-term mobility with weekly subcutaneous Burosumab injections. In future it is expected targeted therapy in conjunction with continued multi-disciplinary involvement will have a major impact on morbidity in patients living with X-linked Hypophosphataemic Rickets.

\section{P40 HYPERCALCAEMIA IN A CHILD}

${ }^{1}$ Lubna Mahmood*, ${ }^{2}$ Michelle Dillon. 'St. Luke's Hospital, Kilkenny, Ireland; ${ }^{2}$ St. Luke's Hospital, Kilkenny, Ireland

\subsection{6/archdischild-2019-epa.395}

Introduction Hypercalcaemia is an infrequent finding in children. In adults, causes are most often malignancy or primary hyperparathyroidism. In childhood the aetiologies are diverse, may be age specific and many have an underlying genetic basis.

Presentation Hypercalcaemia may be found as an incidental finding with no associated clinical features. When clinical features are present there has usually been an insidious onset over a few weeks. Most frequent findings are lethargy, hypotonia, anorexia, weight loss or failure to thrive, polydipsia, polyuria, vomiting, bone pain, constipation and abdominal pain. In severe cases, renal failure, pancreatitis and reduced consciousness may occur. Drug history must include enquiry regarding intake of alternative medicines. It is important to ascertain whether other family members have had hypercalcaemia, renal stones, parathyroidectomy or features of multiple endocrine neoplasia syndromes. Examination should assess the degree of dehydration, look for features of malignancy bone pain or vertebral fractures and the presence of a rash. Evidence of dysmorphism or skeletal disproportion should also be sought.

Case report 15 months old girl was reviewed in Paediatrics outpatient with mild gross motor delay, fussy eating, constipation, intermittent episodes of screaming and poor sleep pattern. Serum Calcium was noted to be elevated at 2.81, repeat few weeks later remained same. Further workup was done. Bone profile normal, PTH normal, Vitamin D increase risk of inadequacy. Metabolic workup normal. She was commenced on IV fluids and oral Furosemide to bring Calcium down and later discharged home on oral Furosemide and low Calcium diet. Urinary Cacium was noted to be low. Father's results 
showed a high Calcium of $2.89 \mathrm{mmol} / \mathrm{L}$ with a low urine Calcium supporting the likely diagnosis of familial hypocalciuric hypercalcaemia. Molecular analysis of AP2S1 gene was abnormal. This result confirmed a molecular disgnosis of $\mathrm{FHH}$ type 3.

Discussion $\mathrm{FHH}$ is an autosomal dominant condition. FHH1 is caused by loss of function in CASR gene, FHH2 by a mutation in GNA11 gene and FHH3 identified as mutation in AP2S1 gene. Mutations lead to an elevation of the normal set point for maintaining normal plasma calcium levels. This causes mild to moderate hypercalcaemia with ionised calcium levels usually within $10 \%$ of the upper limit of normal. Urine calcium/creatinine ratio tends to be low in $\mathrm{FHH}$ whereas it is usually elevated in primary hyperparathyroidism. In the classic description of $\mathrm{FHH}$, individuals are asymptomatic with normal bone density and no intervention is usually required.

\section{P41 NOT YOUR TYPICAL RICKETS CASE}

Rachel Beckett*, Emmeline Heffernan. Royal Belfast Hospital for Sick Children, Belfast, UK

\subsection{6/archdischild-2019-epa.396}

Presentation A healthy caucasian 3 year old girl was referred due to bowing of her femora, apparent since she started walking at 13 months. She was reported to be clumsy and tire easily. There was no history of fractures or leg pain. Her height had dropped from the $75^{\text {th }}$ centile to between the $25^{\text {th }}$ and $50^{\text {th }}$ centile. Initial investigations showed mildly low corrected calcium and phosphate, slightly raised alkaline phosphatase and a sufficient vitamin $\mathrm{D}$ level of $50 \mathrm{nmol} / \mathrm{L}$. She was treated with oral Vitamin D supplements. X-ray of knees was normal. On review after 6 months, bowing had progressed and height had fallen further. This led to the consideration of rarer forms of rickets.

Further investigations Urinary calcium creatinine ratio was normal at 0.07 but urinary phosphate: creatinine ratio was elevated at 4.36; with reduced tubular reabsorption of phosphate - in keeping with a diagnosis of $\times$ linked hypophosphataemic rickets. This was confirmed by detection of a mutation in the PHEX gene.

Skeletal survey showed lower limb abnormalities, including flared metaphyses, widening of the growth plates and buttressing of the femur and tibia. Renal ultrasound showed no nephrocalcinosis. Parental blood tests showed a slightly low phosphate level in her mother.

Progress and treatment She was treated with oral phosphate supplements and alfacalcidol. Since commencing treatment her growth has improved and she remains active but still tires easily. She was subsequently diagnosed with moderate sensorineural hearing loss, another feature of hypophosphataemic rickets, and has had bilateral hearing aids fitted.

$\mathrm{X}$ linked hypophosphataemic rickets Rickets is a disorder of the growth plate, due to inadequate supply of phosphate to growing bones. Mutations in the PHEX gene cause increased levels of fibroblast growth factor 23 (FGF23), resulting in reduced absorption of phosphate in the proximal renal tubule. Although rare, it is the most common form of hereditary rickets, and usually presents in the first 2 years of life. Investigations show low serum phosphate and the key feature is significant phosphaturia (calculated by $\mathrm{TmP} / \mathrm{GFR}$ ). Patients are at increased risk of dental complications, enthesopathy, lumbar lordosis and hearing impairment. Phosphate supplements replace renal losses and calcitriol increases phosphate absorption from the gut and reduces PTH, preventing nephrocalcinosis. A new treatment, Burosumab is a monoclonal IgG1 antibody that binds excess FGF23. This normalises phosphate levels and improves bone mineralisation.

\section{P42 SEPTIC ARTHRITIS OF ELBOW JOINT IN AN 11 MONTH BABY FOLLOWING CHICKENPOX, A CASE REPORT}

Mohamed Elrih*, Ali Omer, Mohamed Shalan, Mr William Gaine. Sligo University Hospital, Sligo, Ireland

\subsection{6/archdischild-2019-epa.397}

An 11 month baby girl presented with 1 week history of left elbow pain and swelling with restricted mobility, on and off fever treated with paracetamol at home. 3 weeks prior to her presentation she had chickenpox with generalized skin rashes. Baby has no past medical history and up to date with her vaccinations. Clinically elbow was swollen with redness at the dorsal lateral side mainly, very tender to touch with almost no range of motion at a slight flexed position. Routine bloods showed significant increase in the inflammatory markers, ultra-sound of the elbow show moderate joint effusion with fluid in the anterior and posterior aspects of the joint with small foci of debris within the effusion, Synovial thickening and increased vascularity with no irregularity of the underlying bony contours. As simple aspiration was difficult to be performed given her age, patient was taken to theater and urgent drainage on the day of presentation was done, with about $10-15 \mathrm{ml}$ of yellowish discharge drained from the lateral side of the elbow. Blood culture along with the joint fluid showed growth of Streptococcus Pyogenes which was sensitive to Penicillin. After 4 weeks of Intravenous Penicillin and another 4 weeks of oral Amoxicillin, baby showed complete recovery both clinically and on her routine bloods.

\section{P43 THE POWER OF THE MIND}

${ }^{1}$ Cathleen McCarrick*, ${ }^{1}$ Noelle Cassidy, ${ }^{2}$ Karen Kelleher. ${ }^{1}$ Temple Street, Children's University Hospital, Dublin, Ireland; '2Our Lady's Children's Hospital, Crumlin, Dublin, Ireland

\subsection{6/archdischild-2019-epa.398}

Introduction Somatoform disorders are a challenge for all branches within medicine but particularly for those working within the paediatric sphere. They are characterized by physical symptoms that are inconsistent with or not fully explained by any underlying medical or surgical diagnosis. In order to reach the diagnosis of a somatoform disorder, children are typically subjected to a number of investigations.

Somatoform disorders can have a significant impact within two domains; the economic impact on healthcare resources in reaching a diagnosis and the effect on children in terms of the underlying emotional stress, the practical impact on families, school absenteeism and the treatment of such a condition. For the purposes of this study, we examined the incidence of somatoform disorders within an orthopaedic cohort of patients. 